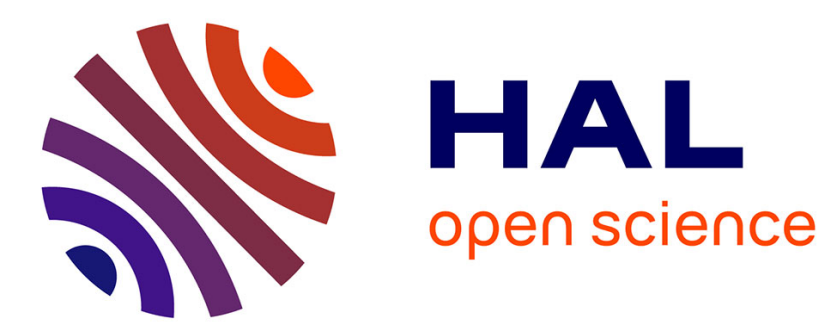

\title{
X-ray spontaneous emission control by 1-dimensional photonic bandgap structure
}

Jean-Michel André, Philippe Jonnard

\section{To cite this version:}

Jean-Michel André, Philippe Jonnard. X-ray spontaneous emission control by 1-dimensional photonic bandgap structure. The European Physical Journal D : Atomic, molecular, optical and plasma physics, 2010, 57, pp.411. 10.1140/epjd/e2010-00050-7 . hal-00596495

\section{HAL Id: hal-00596495 \\ https://hal.science/hal-00596495}

Submitted on 27 May 2011

HAL is a multi-disciplinary open access archive for the deposit and dissemination of scientific research documents, whether they are published or not. The documents may come from teaching and research institutions in France or abroad, or from public or private research centers.
L'archive ouverte pluridisciplinaire HAL, est destinée au dépôt et à la diffusion de documents scientifiques de niveau recherche, publiés ou non, émanant des établissements d'enseignement et de recherche français ou étrangers, des laboratoires publics ou privés. 


\title{
X-ray spontaneous emission control by 1-dimensional photonic bandgap structure
}

Jean-Michel André and Philippe Jonnard

Laboratoire de Chimie Physique - Matière et Rayonnement, UPMC Univ Paris 06, 11 rue Pierre et Marie Curie, F-75231 Paris Cedex 05, France

CNRS - UMR 7614, 11 rue Pierre et Marie Curie, F-75231 Paris Cedex 05, France

\begin{abstract}
The possibility of controlling the x-ray spontaneous emission of atoms embedded in a 1dimensional photonic bandgap structure by the so-called Purcell effect, is studied. Calculations of the spontaneously emitted power are presented from Fermi's golden rule in the framework of the Wigner-time approach extended to absorbing media. Numerical simulations are compared to experimental results for the case of the $\mathrm{K}$ emission from silicon atoms excited by electrons within a $\mathrm{Mo} / \mathrm{Si}$ multilayer Bragg reflector. The inhibition or enhancement of $\mathrm{x}$-ray emission from such structures appear to be feasible.
\end{abstract}

\section{PACS}

42.25.Bs Wave propagation, transmission and absorption

42.70.Qs Photonic bandgap materials

78.20.-e Optical properties of bulk materials and thin films

78.67.Pt Multilayers; superlattices; photonic structures; metamaterials

78.70.En X-ray emission spectra and fluorescence

Corresponding author : Dr. Jean-Michel André

Laboratoire de Chimie Physique - Matière et Rayonnement, 11 rue Pierre et Marie Curie, F-75231 Paris Cedex 05, France

Tel : $33144276614 \quad$ fax : 33144276226

Jean-michel.andre1@upmc.fr 


\section{INTRODUCTION}

Alteration of the spontaneous atomic emission rate by non-trivial boundary conditions on the radiation was pointed out in a classic paper by Purcell [1]. From Fermi's golden rule it appears that this rate is proportional to the density of mode (DOM) of the radiation in the medium. Model of this effect valid in the Wigner-Weisskopf regime, has been done in a classical formalism [2,3]. The advent of Photonic Band-Gap (PGB) structures has permitted to manipulate the DOM and then to control the spontaneous emission (SE) of atoms. Both enhancement and inhibition have been performed in the long wavelength domains by means of a one-dimensional PGB (1D-PGB) structure equivalent to a distributed Bragg reflector (DBR): it was possible to enhance the emission provided that the emission frequency of the atomic transition coincides with the frequencies of the edges of the band-gap and to stop it if the emission frequency of the atomic transition is located inside the band-gap [4,5]. Indeed the DOM of a DBR may present important peaks at the band-edges that allow the high extraction efficiency of the emission. The ability to control the spontaneous emission has profound consequence on many optoelectronics devices [6].

On another side, the progresses in the techniques of thin film deposition have permitted to fabricate periodic multilayered stack on nanometric scale that work as efficient Bragg reflector in the short wavelength domain of the electromagnetic spectrum (uv and x-rays) [7,8]. Then it appeared that these structures can be implemented to monitor the atomic emission in this spectral region. Thus Kossel-like structures have been observed under electron excitation in several Bragg mirrors for the $\mathrm{M} \alpha$ emission of tungsten in $\mathrm{W} / \mathrm{C}$ multilayers [9,10] or the K $\alpha$ emission of silicon in $\mathrm{Mo} / \mathrm{Si}$ multilayers [11]. These works were, to our knowledge, the first experimental evidence of the possibility to enhance atomic SE in the $\mathrm{x}$-ray domain by means of a 1D-PBG. The implementation of 1D-PBG as Distributed Bragg Reflector in the $\mathrm{x}$-ray domain should play an important role in the development of $\mathrm{x}$ ray laser [12] as emphasized by Yariv and Yeh.

In this paper, the feasibility of controlling the SE in the x-ray domain by using Bragg multilayer structure is studied through the concept of DOM. First we give the formula deduced from Fermi's golden rule to calculate the SE rate from the DOM and the normal modes (NM). Then we explain how to compute the DOM in the Wigner time approach (WTA) for absorbing media and the NMs for a Bragg reflector. We discuss the feasibility of controlling the SE through numerical examples. Finally the results of the theoretical model are compared to experimental data obtained for the $\mathrm{Si}-\mathrm{K}$ emission line emitted from a $\mathrm{Mo} / \mathrm{Si}$ Bragg reflector under electron excitation. 


\section{SPONTANEOUS EMISSION RATE IN A 1D-PBG STRUCTURE}

The spontaneous emission rate (SER) of an emitting atom is given by Fermi's golden rule :

$$
\tau(R)=\frac{2 \pi}{\hbar^{2}}|\langle f|H| i\rangle|^{2} \delta\left(\omega_{i}-\omega_{f}\right)
$$

in which $|\mathrm{i}\rangle$ and $|\mathrm{f}\rangle$ are the initial and final states of energies $\hbar \omega_{i / f} . \mathrm{H}$ is the interaction Hamiltonian of the whole system including emitting atoms, radiation and the optical «box »; it is given by :

$$
H=-\vec{\mu}(\mathbf{R}) \cdot \vec{E}(\mathbf{R})
$$

where $\vec{\mu}(\mathbf{R})$ and $\vec{E}(\mathbf{R})$ are the dipole moment operator of the atom positioned at $\mathbf{R}$ and the electric field operator respectively. In the Wigner-Weisskopf regime, the mode density is the same classically or quantum electrodynamically; then the SER can be derived from the classical model of a point dipole inside a medium with an inhomogeneous dielectric constant $\varepsilon(\mathbf{r})$. In this approach the emitting atom is modeled by an harmonically oscillating dipole with frequency $\omega_{0}$ and dipole moment $\vec{\mu}$ located at $\mathbf{R}$; the corresponding current density $\mathbf{J}(\mathbf{r}, t)$ to is taken :

$$
\mathbf{J}(\mathbf{r}, t)=\Omega \vec{\mu}(\mathbf{R}) \cos \left(\omega_{0} t\right) \Theta(t) \delta(\mathbf{r}-\mathbf{R})
$$

The steady-state rate of power emission after all transients have vanished is given by:

$$
P(t)=\frac{\pi^{2}}{2} \Omega^{2} \mu^{2} \int\left|\mathbf{a}_{\mathbf{k}}(\mathbf{R}) \cdot \vec{\mu}\right|^{2} \delta\left(\omega_{0}-\omega_{\mathbf{k}}\right) d^{3} k
$$

For sake of consistency the calculation are summarized in the Appendix. By integrating this expression over all wave-numbers $\mathrm{k}$, the DOM $\bar{\rho}(\omega)=\frac{d k}{d \omega}$ appears as a result of changing variables $\mathrm{k}$ to $\omega_{\mathrm{k}}$. It yields the power spectrum $\mathrm{P}_{\omega}$ of the emitted radiation as follows :

$$
P_{\omega}=C \bar{\rho}(\omega)\left|\mathbf{a}_{\mathbf{k}(\omega)}\right|^{2}
$$

where C is some constant. Since The DOM is independent of position inside either structure, the averaging of the power spectrum is effected by replacing the values of $\left|\mathbf{a}_{\mathbf{k}(\omega)}\right|^{2}$ by the corresponding averaged value :

$$
\left\langle\left|\mathbf{a}_{\mathbf{k}(\omega)}(z)\right|^{2}\right\rangle=\frac{1}{Z_{\max }-Z_{\min }} \int_{Z_{\min }}^{Z_{\max }}\left|\mathbf{a}_{\mathbf{k}(\omega)}(z)\right|^{2} d z
$$


The integration is performed along the $\mathrm{z}$-axis in the region occupied by the emitting medium between $\mathrm{z}=\mathrm{Z}_{\min }$ and $\mathrm{z}=\mathrm{Z}_{\max }$.

\section{DOM FOR ABSORBING MEDIA IN THE WIGNER-TIME APPROACH \\ III.1 Matrix transfer formalism for absorbing media}

In the Wigner-time approach the DOM, $\bar{\rho}(\omega)$, is considered to be the reciprocal of the group velocity $\mathrm{v}$ :

$$
\bar{\rho}(\omega)=\frac{1}{v}=\frac{d k(\omega)}{d \omega}
$$

Since the complex transmission coefficient $t(\omega)$ contains phase information from which the dispersion relation $\mathrm{k}(\omega)$ can be extracted, one understands easily that the group velocity $\mathrm{v}$, hence the DOM $\bar{\rho}(\omega)$ can be deduced from $t(\omega)$ as explained in a paper [13] by Bendickson et al. These authors show that :

$$
\bar{\rho}(\omega)=\frac{1}{D} \frac{y^{\prime} x-x^{\prime} y}{x^{2}+y^{2}}
$$

where $\mathrm{D}$ is the total length of the structure, and $\mathrm{x}$ and $\mathrm{y}$ the real and imaginary part of $\mathrm{t}$, respectively; the prime denotes differentiation with respect to $\omega$. It is common to present a dimensionless DOM $\rho(\omega)$ obtained by multiplying $\bar{\rho}(\omega)$ by the group velocity in the bulk medium Vbulk.

Our purpose now is to calculate the transmission coefficient of a N-period Bragg reflector made up with absorbing materials. As usual when dealing with the theory of electromagnetic propagation in linear stratified structure, we apply the transfer matrix formalism. Let us recall that the transfer matrix describes the transfer properties of the stratified system : it relates the amplitude of the incoming and outgoing electric fields and its expression depends on the geometry under consideration. In the Left-To-Right propagation (LTR) case considered in this paper and described in Figure 1, one has :

$$
\left(\begin{array}{l}
1 \\
\vec{r}
\end{array}\right)=\vec{M}\left(\begin{array}{l}
\vec{t} \\
0
\end{array}\right)
$$

with $[14,15]$ :

$$
\overrightarrow{\mathbf{M}}=\left(\begin{array}{cc}
\frac{1}{\vec{t}} & \frac{\vec{r}^{\#}}{\vec{t}^{\#}} \\
\frac{\vec{r}}{\vec{t}} & \frac{1}{\vec{t}^{\#}}
\end{array}\right)
$$


where $\vec{t}$ and $\vec{r}$ are the complex transmission and reflection coefficients of the whole system, respectively. The arrow $\rightarrow$ used as superscript expresses the LTR propagation.

This expression of the matrix transfer is obtained from the linearity of the problem and from the time-reversal symmetry that is the fact that Maxwell's equations are symmetric under time reversal. To describe the time reversal in our context of absorbing media, a special complex conjugation is required. We denote this operation by the superscript \#. It means a standard complex conjugation plus the reversal of all the signs of the imaginary part of the complex optical indices. The requirement to this special conjugation can be justified as follows: in a time reversed process for propagation in absorbing media, the fields are actually amplified; converting absorption to amplification requires the reversal of the sign of the imaginary part of the refractive index. This point is crucial in our calculation. Generally the time reversal for lossless systems is got by the mere complex conjugation [14].

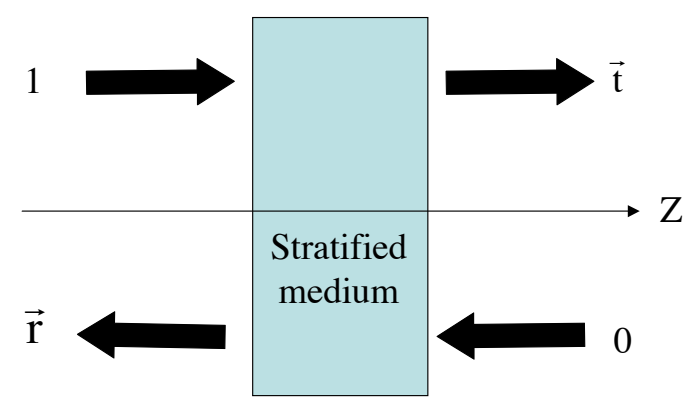

Figure 1: Left-to-Right (LTR) propagation scheme. $\mathrm{Z}$ is the direction of stratification of the medium.

The calculation of the DOM of absorbing composite quasi-periodic stratified structure requires the implementation of generalized Stokes reciprocity, where \#-conjugation associated with the time reversal and parity reversal are involved. This generalization is presented in Ref. [15]. For convenience we recall that parity reversal is a process where a field of unit amplitude incident from the right gives rise to new Right-To-Left (RTL) complex transmission and reflection coefficients $\overline{\mathrm{t}}$ and $\overline{\mathrm{r}}$, respectively. The RTL process is displayed in Figure 2. 


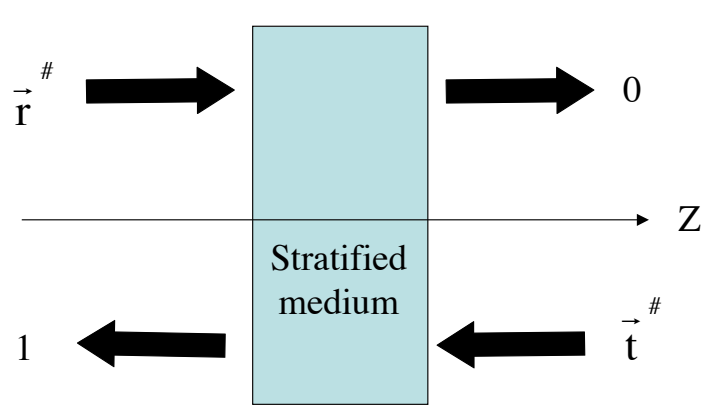

Figure 2: Right-To-Left (RTL) propagation scheme.

\section{III.2 DOM of a N-period Bragg reflector}

The N-period Bragg reflector is made of a stack of $\mathrm{N}$ bilayers with two materials denoted by 1 and 2 . The LTR transfer matrix of the N-period system can be expressed by means of the Chebychev polynomials of second kind $\mathrm{U}_{\mathrm{N}}(\mathrm{x})$ and of the coefficients $\mathrm{C}_{\mathrm{ij}}$ of the bilayer transfer matrix as follows [16] :

$$
\overrightarrow{\mathbf{M}}=\left(\begin{array}{cc}
C_{11} U_{N-1}(K d)-U_{N-2}(K d) & C_{12} U_{N-1}(K d) \\
C_{21} U_{N-1}(K d) & C_{22} U_{N-1}(K d)-U_{N-2}(K d)
\end{array}\right)
$$

where

$$
\begin{aligned}
& C_{11}=\frac{1}{1-r_{1,2}^{2}}\left(\exp (i \Phi)-r_{1,2}^{2} \exp (-i \Delta)\right) \\
& C_{22}=\frac{1}{1-r_{1,2}^{2}}\left(\exp (-i \Phi)-r_{1,2}^{2} \exp (i \Delta)\right) \\
& C_{12}=\frac{r_{1,2}}{1-r_{1,2}^{2}}(\exp (-i \Phi)-\exp (i \Delta)) \\
& C_{21}=\frac{r_{1,2}}{1-r_{1,2}^{2}}(\exp (i \Phi)-\exp (-i \Delta))
\end{aligned}
$$

with

$$
\Phi=k_{\perp 1} d_{1}+k_{\perp 2} d_{2} \text { and } \Delta=k_{\perp 1} d_{1}-k_{\perp 2} d_{2}
$$

The term $\mathrm{k}_{\perp \mathrm{j}}$ is the perpendicular component of the wave-vector in the layer $\mathrm{j}=1,2$. It obeys the Snell's law. The coefficient $r_{1,2}$ is the Fresnel coefficient associated with the reflection at the interface between the layer 1 and the layer 2 ; its expression is given by the Fresnel formula $[16,17] . \mathrm{K}$ is the so-called Bloch-Floquet wave-number; it corresponds to the wave-number associated with an infinite periodic system, i.e. where $\mathrm{N} \rightarrow \infty$. In fact the main problem in the determination of the transfer matrix comes from the calculation of K. Different 
ways can be used to calculate it $[16,18]$. It can be obtained from the trace $\mathrm{S}$ of the bilayer transfer matrix as :

$$
K=\frac{1}{d} \cos ^{-1}(S)=\frac{1}{d} \cos ^{-1}\left(C_{11}+C_{22}\right)
$$

The transmission coefficient $\vec{t}(\omega)$ and the reflection coefficient $\vec{r}(\omega)$ can be deduced by identification of Eqs. (10) and (11). Thus,

$$
\vec{t}(\omega)=\left(C_{11} U_{N-1}(K d)-U_{N-2}(K d)\right)^{-1}
$$

and

$$
\vec{r}(\omega)=\vec{t}(\omega) C_{21} U_{N-1}(K d)
$$

\section{III.3 Numerical for a Mo/Si Bragg reflector with $\mathbf{N}$ periods}

The thickness $d$ of the bi-layer is obtained from the Bragg condition :

$$
d=\frac{c p \pi}{\bar{\omega}_{r} \sin \theta_{r}}
$$

where $\bar{\omega}_{\mathrm{r}}$ is the Bragg frequency, $\theta_{\mathrm{r}}$ the Bragg angle and $\mathrm{p}$ the order of diffraction. We shall see that the value of the Bragg frequency and of the Bragg angle depends on the problem that one has to solve. If one wishes to obtain an inhibition of the emission corresponding to the resonant frequency $\omega_{0}, \bar{\omega}_{\mathrm{r}}$ will be chosen to be equal to $\omega_{0}$ so that the resonant frequency lies in the forbidden gap. On the contrary if one wishes to obtain an exaltation of the emission at resonant frequency $\omega_{0}, \bar{\omega}_{\mathrm{r}}$ will be chosen so that the frequencies $\omega^{+}$and $\omega^{-}$of the band-edges coincide with $\omega_{0}$; the choice is not obvious and can be done from a a priori estimation of the bandwidth $\Delta \omega$; a strategy to reach this goal will be presented hereafter.

As an example, we consider a molybdenum (Mo) and silicon (Si) Bragg reflector. This $\mathrm{Mo} / \mathrm{Si}$ system is standard in the soft-X-ray domain as Bragg mirrors. We deal with the Silicon $\mathrm{K}(\mathrm{Si}-\mathrm{K})$ emission, which means the Bragg reflector is designed to be able to diffract at the energy $E_{0}=1740 \mathrm{eV}$, corresponding to the resonant frequency $\omega_{0}=264310^{15} \mathrm{~s}^{-1}$; as explained previously one obtains the period $d$ by applying the condition (15) with $\bar{\omega}_{\mathrm{r}}=\omega_{0}$. One has $d=4.8 \mathrm{~nm}$ for a Bragg angle $\theta_{\mathrm{r}}=4.26^{\circ}$.

Figure 3 shows the DOM $\bar{\rho}(\Omega)$ and the transmittance T versus the reduced frequency $\Omega=\omega / \omega_{0}$, calculated in the WTA for the Bragg angle $\theta_{\mathrm{r}}=4.26^{\circ}$, a duty cycle $\gamma$ (ratio of the Mo layer thickness $d_{1}$ to the bilayer thickness $d$ ) of 0.33 and for different values of the number of bilayers $\mathrm{N}$. The calculations are performed for the $s$-polarization case and we use 
the following values for the complex optical index $[16,19]: \mathrm{n}_{\mathrm{Mo}}=1-0.01316-\mathrm{i} 0.00289$ and $\mathrm{n}_{\mathrm{Si}}=1-0.00252-\mathrm{i} 0.00055$.

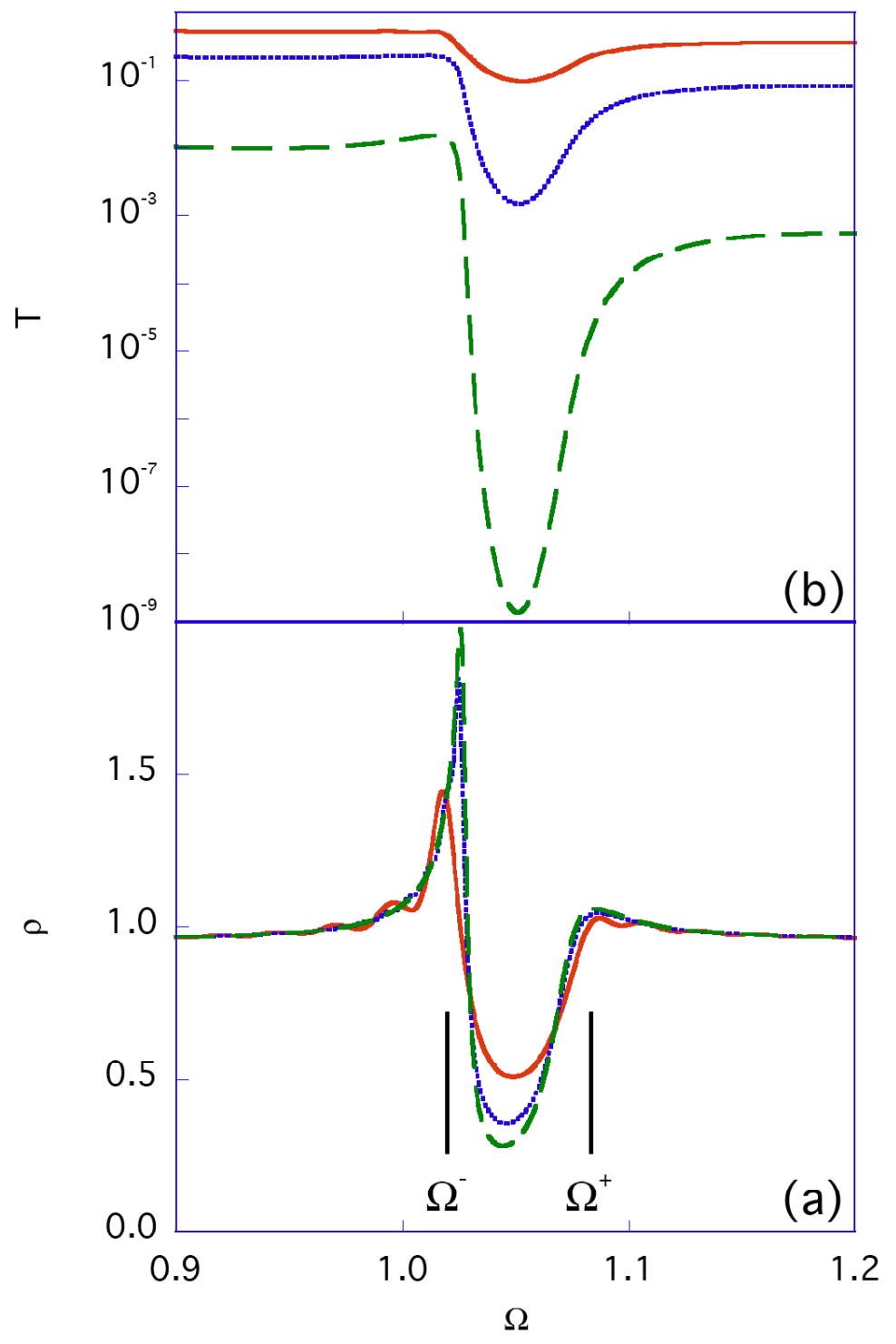

Figure 3: Calculation taking into account the absorption of the DOM (a) and the transmittance (b) of a Mo/Si Bragg reflector versus the reduced frequency as a function of the number of bilayers: 40 (solid line), 100 (dotted line) and 300 (dashed line).

As expected, one observes peaks at the band-edges $\left(\Omega^{-}, \Omega^{+}\right)$but the peak corresponding to the reduced frequency $\Omega^{-}$is more intense than the other one at the reduced frequency $\Omega^{+}$. This effect corresponds to an asymmetry in the transmittance and arises from the occurrence of absorption in the materials. Figure 4 shows that quasi-symmetry is obtained in the noabsorption case in the case of a Bragg reflector made of $100 \mathrm{Mo} / \mathrm{Si}$ bilayers. It is observed that the curves with and without absorption are slightly shifted. This is probably due to the fact that the calculations are made without taking into account the variation of the indices as a function of the wavelength. When the number $\mathrm{N}$ increases, the peak at the band-edge $\Omega^{-}$ 
becomes narrower and more intense. This result is in agreement with the well-known fact that for an infinite periodic system, the limit of the Brillouin zones in the $(k, \omega)$ domain have a step-like shape and consequently, the DOM $\bar{\rho}(\omega)=\frac{d k(\omega)}{d \omega}$ has the behavior of a Dirac delta function. The $\Omega$-bandwidth $\Delta \Omega$ is equal to 0.062 which means that the spectral width of the photonic band-gap $\triangle \mathrm{E}_{\mathrm{PBG}}$ is close to $102 \mathrm{eV}$. Considering that the natural width of the $\mathrm{Si}-\mathrm{K}$ line is close to $0.5 \mathrm{eV}$ [20], that is much narrower than $\Delta \mathrm{E}_{\mathrm{PBG}}$, the control of the $\mathrm{SE}$ by matching the frequency $\omega_{0}$ of the SE to the DOM shape seems feasible. Practically the tuning can be obtained by varying the $d$-spacing or the Bragg angle.

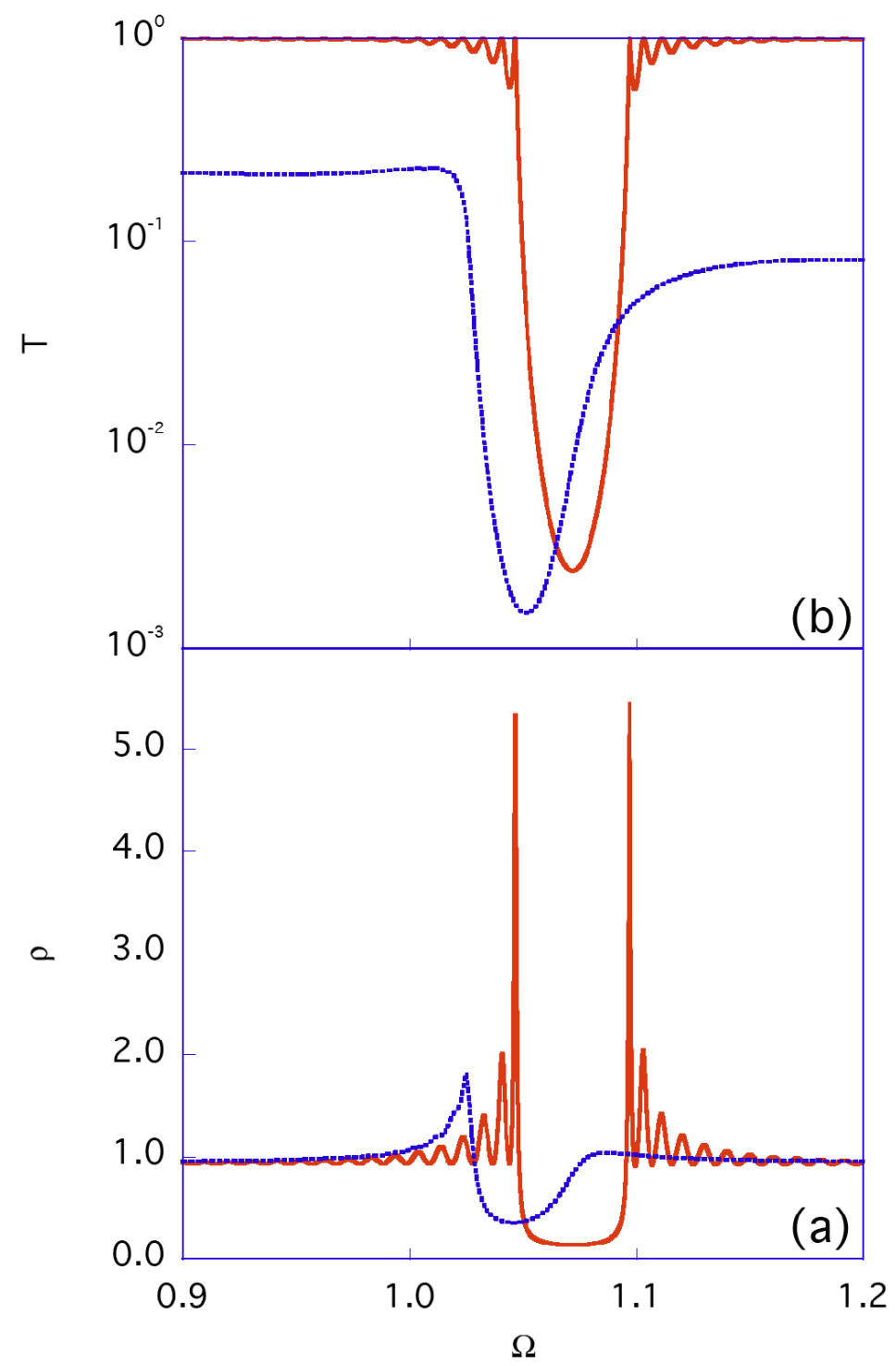

Figure 4: Calculation with (dotted line) and without (solid line) absorption of the DOM (a) and the transmittance (b) of a Mo/Si Bragg reflector with 100 bilayers versus the reduced frequency. 


\section{CHOICE OF THE PERIOD FOR INHIBITION AND ENHANCEMENT OF THE SPONTANEOUS EMISSION}

\section{IV.1 Inhibition}

The goal is to inhibit the SE of frequency $\omega_{0}$ (energy $\mathrm{E}_{0}$ ) by locating this frequency between the band-edge $\omega^{-}$(or $\omega^{+}$if absorbing is very small) of the DOM. In this case, one may fix the Bragg frequency $\bar{\omega}_{\mathrm{r}}$ that is located practically in the middle of the bandgap (i.e. at $\left.\omega+\Delta \omega / 2=\omega^{+}-\Delta \omega / 2\right)$ just at the resonant frequency $\omega_{0}$; the choice of Bragg angle $\theta_{\mathrm{r}}$ is generally governed by practical considerations (geometry of the experiment, minimum practical value of the $d$-spacing, ...). Once $\bar{\omega}_{\mathrm{r}}$ and $\theta_{\mathrm{r}}$ are given, one obtains $d$ from Eq. (15). Let us note that in the soft-x-ray domain where the effect of refraction can be important, it is valuable to implement of Bragg law that takes into account this effect; for further details, see for instance [16].

\section{IV.2 Enhancement}

This case is more difficult to deal with than the previous one. Indeed to get enhancement it is necessary to locate the resonant frequency $\omega_{0}$ of the SE at one of the bandedge frequencies $\omega^{+}$or $\omega^{-}$; the problem is that the value of these frequencies depends on the value of the $d$-spacing. Thus, it is useful to have an approximate value of the bandwidth $\Delta \omega$ to deduce an approximate value of the band-edge frequencies from the relationship :

$$
\omega^{ \pm}=\omega_{r} \pm \frac{\Delta \omega}{2}
$$

The two possible values $d^{+}$and $d^{-}$of the $d$-spacing are then obtained by combining Eqs. (15) and (16) :

$$
d^{ \pm}=\frac{\mathrm{cp} \pi}{\left(\omega_{0} \mp \frac{\Delta \omega}{2}\right) \sin \theta_{\mathrm{r}}}
$$

In absence of absorption, analytical expressions are available to estimate $\Delta \omega$. From Eq. (A.8) of Ref. [16], one deduces that the following equation can be used to estimate the bandwidth :

$$
\Delta \omega \approx 4 \gamma\left|\vec{r}\left(\omega_{0}\right)\right||\sin c(p \gamma \pi)| \omega_{0}
$$

where sinc denotes the cardinal sine function, $\sin (\mathrm{x}) / \mathrm{x}$. 


\section{CALCULATION OF THE NORMAL MODES}

\section{V.1 Generalities}

Let us recall that the normal modes $a_{\mathbf{k}}(\mathbf{r})$ can be defined from the harmonic solution :

$$
A_{\mathbf{k}}(\mathbf{r}, t)=a_{\mathbf{k}}(\mathbf{r}) \exp \left(-i \omega_{\mathbf{k}} t\right)
$$

of the homogeneous form (i.e. in absence of current) of the wave equation for the vector potential $\mathbf{A}(\mathbf{r}, \mathrm{t})$ :

$$
\nabla \times \nabla \times \mathbf{A}(\mathbf{r}, t)+\frac{1}{c^{2}} \varepsilon(r) \ddot{\mathbf{A}}(\mathbf{r}, t)=\mathbf{0}
$$

in the transverse jauge condition. In fact since our problem is one-dimensional (1D), the 1D $\mathrm{NM} \mathrm{a}_{\mathrm{k}}(\mathrm{z})$ obeys the Helmholtz equation :

$$
a_{\mathbf{k}}{ }^{\prime \prime}(z)+\frac{\omega_{\mathbf{k}}^{2}}{c^{2}} \varepsilon(z) a_{\mathbf{k}}(z)=0
$$

and must obey a completeness relationship of the form :

$$
\int a_{\mathbf{k}}^{*}(z) a_{\mathbf{k}}\left(z_{0}\right) d \mathbf{k}=\delta_{t}\left(z-z_{0}\right)
$$

where $\delta_{\mathrm{t}}$ stands for the $\varepsilon$-transverse Dirac function. They must also fulfill the boundary conditions, that is, their continuity and the continuity of their derivative with respect to the space variable $\mathrm{z}$ at the boundary as a result of the continuity of the parallel components of the magnetic and electric fields.

\section{V.2 Case of the N-period Bragg reflector}

Let us now consider the two homogeneous regions denoted 1 and 2 of optical indices $n_{1}$ and $\mathrm{n}_{2}$ respectively, forming the unit cell of the stratified medium, that is the bilayer in a Bragg reflector. In each of the region, the NM consists of a superposition of Left- and Rightgoing waves of wave-vector normal component $\mathrm{k}_{\perp \mathrm{j}}, \mathrm{j}=1$ or 2 as shown in figures 1 and 2 . Hence, one writes in the first unit cell with index $n=0$ :

$$
a_{j}^{(n=0)}(z)=A_{j} \exp \left(i k_{\perp j} z\right)+B_{j} \exp \left(-i k_{\perp j} z\right)
$$

By virtue of the Bloch-Floquet theorem, it follows that the form of the mode in the p-th unit cell $(n=p)$ can be generated from the one in the first unit cell $(n=0)$ by :

$$
\begin{aligned}
& a_{j}^{(n=p)}(z)=\exp (i p K d)\left[A_{j} \exp \left(i k_{\perp j}(z-p d)\right)+B j \exp \left(-i k_{\perp j}(z-p d)\right)\right], \\
& \quad p d<z<(p+1) d
\end{aligned}
$$

where $\mathrm{K}$ is the Bloch wave-number corresponding to the overall solution $\mathrm{a}_{\mathrm{K}}(\mathrm{z})$ for a infinite structure $(\mathrm{N} \rightarrow \infty)$. This wave-number will be determined as shown hereafter. 
The continuity condition of the mode and its first derivative denoted by prime at $\mathrm{z}=\mathrm{pd}$ and $\mathrm{z}=\mathrm{d}(\mathrm{p}+\gamma)$ leads to:

$$
\begin{aligned}
& a_{2}^{(n=p)}(z=p d)=a_{1}^{(n=p+1)}(z=p d) \\
& a_{2}^{\prime(n=p)}(z=p d)=a_{1}^{\prime(n=p+1)}(z=p d) \\
& a_{1}^{(n=p+1)}(z=d(p+\gamma))=a_{2}^{(n=p+1)}(z=d(p+\gamma))
\end{aligned}
$$

and

$$
a_{1}^{\prime(n=p+1)}(z=d(p+\gamma))=a_{2}^{\prime(n=p+1)}(z=d(p+\gamma))
$$

By inserting the solutions Eq. (24) in Eqs. (25-28), it follows that the four coefficients $A_{j}$ and $B j(j=1,2)$ obey the following $4 \times 4$ matrix equation :

$$
\hat{\mathbf{M}}\left(K, k_{1}, k_{2}, d, \gamma\right)\left(\begin{array}{l}
A_{1} \\
B_{1} \\
A_{2} \\
B_{2}
\end{array}\right)=\left(\begin{array}{l}
0 \\
0 \\
0 \\
0
\end{array}\right)
$$

where the subscript $\perp$ has been removed for convenience, with :

$$
\hat{\mathbf{M}}\left(K, k_{1}, k_{2}, d, \gamma\right)=\left(\begin{array}{cccc}
\exp \left(i \Gamma_{1}^{+} d\right) & \exp \left(i \Gamma_{1}^{-} d\right) & -1 & -1 \\
i k_{1} \exp \left(i \Gamma_{1}^{+} d\right) & -i k_{1} \exp \left(i \Gamma_{1}^{-} d\right) & -i k_{2} & i k_{2} \\
-\exp \left(i k_{1} \gamma d\right) & -\exp \left(-i k_{1} \gamma d\right) & \exp \left(i k_{2} \gamma d\right) & \exp \left(-i k_{2} \gamma d\right) \\
-i k_{1} \exp \left(i k_{1} \gamma d\right) & i k_{1} \exp \left(-i k_{1} \gamma d\right) & i k_{2} \exp \left(i k_{2} \gamma d\right) & -i k_{2} \exp \left(-i k_{2} \gamma d\right)
\end{array}\right)
$$

where $\Gamma_{j}^{+/-}=K \pm k j$.

The condition for obtaining a nontrivial solution of Eq. (30) is that :

$$
\operatorname{det}\left[\hat{\mathbf{M}}\left(K, k_{1}, k_{2}, d, \gamma\right)\right]=0
$$

that is,

$$
\cos (K d)=-\frac{\left(k_{1}-k_{2}\right)^{2}}{4 k_{1} k_{2}} \cos \left[d\left(k_{1}(\gamma-1)+k_{2} \gamma\right)\right]+\frac{\left(k_{1}+k_{2}\right)^{2}}{4 k_{1} k_{2}} \cos \left[d\left(k_{1}(1-\gamma)+k_{2} \gamma\right)\right]
$$

Eq. (32) is the dispersion relationship whose solution gives the value of the Bloch wavenumber K. To obtain fully the expression of the mode in the $\mathrm{n}$-th cell, one must determine the four coefficients: $A_{1}, B_{1}, A_{2}$ and $B_{2}$. This can be done by calculating the kernel of the submatrix $\hat{M}\left(K, k_{1}, k_{2}, d, \gamma\right)$. One gets :

$$
\begin{aligned}
& A_{1}=2 k_{2}-\exp \left(i d \Gamma_{1}^{-}\right)\left[k_{-} \exp \left(i d \gamma k_{+}\right)+k_{+} \exp \left(i d \gamma k_{-}\right)\right] \\
& A_{2}=\exp \left(-i d \gamma k_{-}\right)\left[k_{-} \exp \left(i d \Gamma_{1}^{+}\right)+k_{+} \exp \left[i d\left(\Gamma_{1}^{+}+2 \gamma k_{2}\right)\right]-2 k_{2} \exp \left(i d \gamma k_{+}\right)\right]
\end{aligned}
$$




$$
\begin{aligned}
& B_{1}=\exp \left(-i d \gamma k_{2}\right)\left\{k_{+} \exp \left[i d\left(\Gamma_{1}^{+}+2 \gamma k_{2}\right)\right]-2 k_{1} \exp \left[i d\left(2 K+\gamma k_{1}\right)\right]-k_{-} \exp \left[i d\left(\Gamma_{1}^{-}+\gamma\left(2 k_{1}+k_{2}\right)\right)\right]\right\} \\
& B_{2}=2 k_{1} \exp \left[i d\left(2 K+\gamma k_{+}\right)\right]+k_{-} \exp \left(i d \Gamma_{1}^{+}\right)-k_{+} \exp \left[i d\left(\Gamma_{1}^{-}+2 \gamma k_{1}\right)\right]
\end{aligned}
$$

with

$$
k_{-}=k_{2}-k_{1} \text { and } k_{+}=k_{2}+k_{1}
$$

These results are a generalization of the Kronig-Penney model [14].

\section{V.3 Kronig-Penney model}

By vanishing the ratio $\gamma(\gamma \rightarrow 0)$, one obtains the results corresponding the KronigPenney (KP) model; let us recall that in this model the thickness of the layer 1 tends to zero and the optical potential is given by a Dirac comb.

From Eqs. (33-37) one obtains the coefficients $\mathrm{A}^{\mathrm{KP}}$ and $\mathrm{B}^{\mathrm{KP}}$ for the KP system :

$$
\begin{aligned}
& A_{1}^{K P}=2 k_{2}\left(1-\exp \left(i d \Gamma_{1}^{-}\right)\right) \\
& B_{1}^{K P}=2 k_{2}\left(\exp \left(i d \Gamma_{1}^{+}\right)-1\right) \\
& A_{2}^{K P}=k_{+} \exp \left(i d \Gamma_{1}^{+}\right)-k_{-} \exp \left(i d \Gamma_{1}^{-}\right)-2 k_{1} \exp (2 i d K) \\
& B_{2}^{K P}=2 k_{1} \exp (2 i d K)+k_{-} \exp \left(i d \Gamma_{1}^{+}\right)-k_{+} \exp \left(i d \Gamma_{1}^{-}\right)
\end{aligned}
$$

in agreement with the results of Ref. [14]. The KP case for an infinite PBG, named "Diraccomb superlattice" has been studied in details for both TE and TM polarization in [21] and $[22]$.

\section{POWER SPECTRUM IN THE INHIBITION AND ENHANCEMENT CASES}

We have calculated from Eq. (5) the power spectrum versus the reduced frequency $\Omega$ of the Si K emission coming from the Mo/Si multilayer system under study in $\S$. III.3. The number of bilayers is 40 . The power is normalized so that, far away from the band-gap, it corresponds to the free-space power, equal to unity. This normalized power is plotted in Figure 5. For inhibition, $\Omega=1$ have to be situated within the band-gap, which means that the propagation of the radiation at the frequency $\omega_{0}$ of the SE is forbidden. For enhancement, one obtains quite similar figures but with an offset of the abscissa scale : due to the absorption the enhancement cannot be achieved at the upper band-edge $\left(\Omega^{+}\right)$but only at the lower band-edge $\left(\Omega^{-}\right)$. Assuming a Bragg angle equal to $4.26^{\circ}$, inhibition can be obtained for $\mathrm{d}=4.8 \mathrm{~nm}$, while for enhancement at $\Omega^{+}, d=d^{+}=4.65 \mathrm{~nm}$, and for enhancement at $\Omega^{-}, d=d^{-}=4.95 \mathrm{~nm}$. One notes that these different cases are achieved for values of $d$-spacing which are different 
enough to be practically monitored ; indeed in the state-of-the-art d-spacing are monitored at better than $0.1 \mathrm{~nm}$.

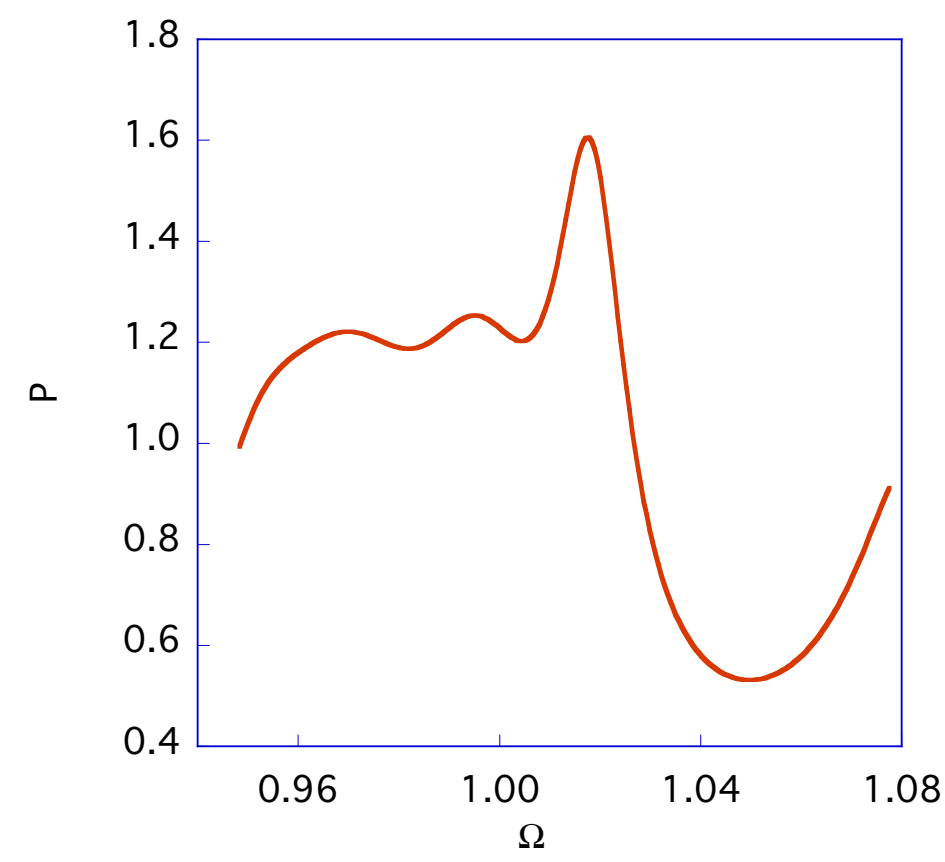

Figure 5: Normalized power of the Si K emission from a Bragg reflector made of $40 \mathrm{Mo} / \mathrm{Si}$ bilayers.

\section{EXPERIMENT}

The details of the experiment can be found in Ref. [11], so we briefly recall its main characteristics. The multilayer is made of $40 \mathrm{Mo} / \mathrm{Si}$ bi-layers, the thickness of the Mo and $\mathrm{Si}$ layers being 1.6 and $3.2 \mathrm{~nm}$ respectively. An electron gun is used to produce the Si $1 \mathrm{~s}$ ionizations necessary for the generation of the $\mathrm{Si} \mathrm{K} \alpha$ emission. The electron energy is $6 \mathrm{keV}$ and in this case all the bi-layers contribute to the emission. The rotation axis of the sample is perpendicular to the plane defined by the directions of the electrons and photons. The sample is rotated around $4.26^{\circ}$, i.e. the Bragg angle of the multilayer at the $\mathrm{Si} \mathrm{K} \alpha$ wavelength $(0.713 \mathrm{~nm})$, with a precision of $0.09^{\circ}$. This angle is between the sample surface and the detection direction and we call it detection angle. During an experiment, the spectrometer, whose acceptance angle is $0.23^{\circ}$, is fixed at the $\mathrm{Si} \mathrm{K} \alpha$ wavelength whose intensity is monitored as a function of the detection angle. The scan is made about $\pm 2^{\circ}$ around the $4.26^{\circ}$ detection angle. It has been verified [11] that a similar experiment performed on a silicon single crystal gives a monotonous behavior as a function of the detection angle and a lower intensity than in the case with the multilayer.

We show in Figure 6 the intensity of the Si K $\alpha$ emission as a function of the detection angle. The angular scale is shifted so that the angle of the maximum is zero. The curve 
increases with the angle until its reaches a maximum. Then a dip occurs, about $0.6^{\circ}$ wide, and afterwards the intensity increases again. This behavior is well reproduced by the calculation of the DOM and the emitted power but on a narrower angular scale : in this case the width of the dip a slightly less than $0.2^{\circ}$ for the DOM and about $0.3^{\circ}$ for the power. The experimental and calculated curves are scaled vertically in order that the intensities of their maximum and minimum coincide. In the power calculation, the in-depth distribution of the Si K ionizations within the multilayer, calculated with a Monte-Carlo code, is used to weight the distribution of the NM in Eq. (6). To take into account the instrumental broadening introduced by the finite angular acceptance of the spectrometer, we apply a $0.23^{\circ}$ moving average on the calculated power. The discrepancy between the width of the experimental and calculated curves is not well understood, but could be ascribed to a bad account of the effect of the acceptance angle of the spectrometer.

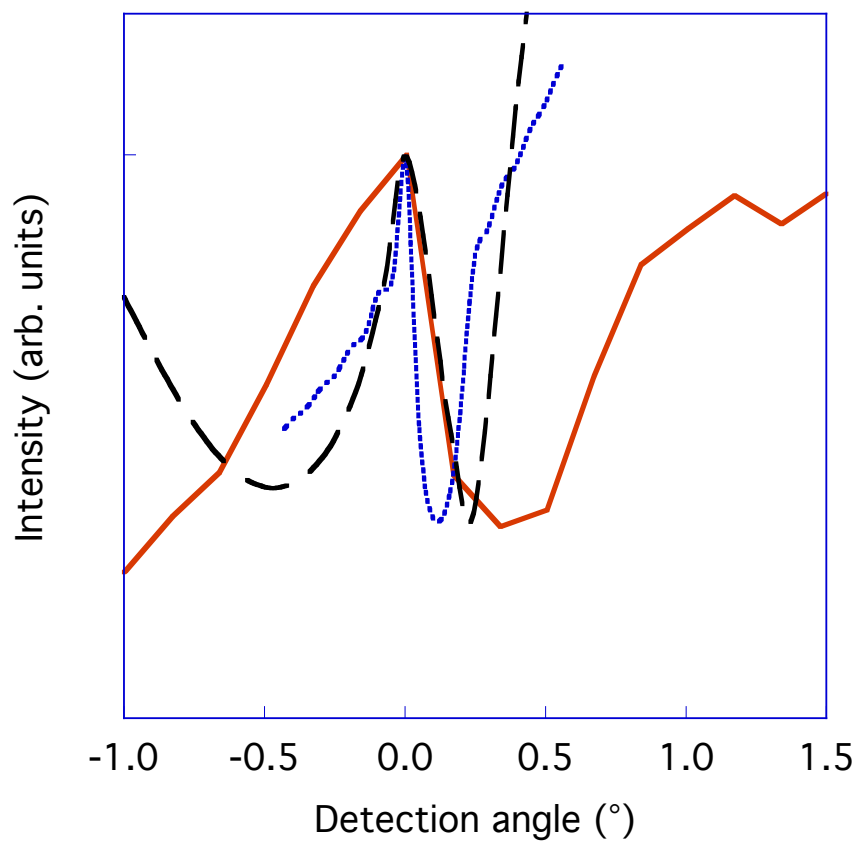

Figure 6: Intensity of the $\mathrm{Si} \mathrm{K} \alpha$ emission coming from a Mo/Si mulilayer excited by $6 \mathrm{keV}$ electrons (solid line) as a function of the detection angle and comparison with the calculation of the DOM (dotted line) and the emitted power broadened by the angular acceptance of the spectrometer (dashed line).

\section{DISCUSSION AND CONCLUSION}

The x-ray SER of a 1D-PBG-embedded emitter can be enhanced provided that the emission energy coincide with the band edge energies of the DOM. On the contrary the spontaneous emission will be partially extinguished if the emission energy is within the forbidden band of the DOM. To obtain enhancement of the SE, one has to center the 
forbidden band on an appropriate energy chosen so that the emission energy coincide with band edge energies. To do it, it is necessary to choose the correct $d$-spacing or detection angle $\theta$, as demonstrated by the presented $\theta$-scan experiment on a Mo/Si multilayer.

Nevertheless one has to keep in mind that the $1 \mathrm{D}-\mathrm{PBG}$ is not a total omnidirectional band gap, which means that a complete suppression or enhancement of the total SE is not possible in all directions with this kind of device. It has been shown that in a high contrast 1D PGB, even in mid-gap emission, the SE can be enhanced parallel to the stack layers where the high-index layers act as resonant waveguides [23]. To achieve overall SE suppression, a full 3D-PBG is required. One has to emphasize that this task is not obvious and evidence of crystals exhibiting a full photonic band-gap has not been reported to date even in the visible light. A step towards an extension of the emission inhibition to further directions is the increase of the "dimensionality" of the PBG. In the x-ray regime, recent development of the so-called lamellar multilayer gratings [24] could offer an opportunity to achieve this goal.

We plan to extend the present study to these gratings both theoretically and experimentally.

The control of the spontaneous emission can also be obtained in more sophisticated systems such as a Fabry-Pérot (FP) resonators with the emitters located within the FP spacer. In this case one can possibly draw advantages from the resonant defect states located within the forbidden band of the DOM $[14,18]$.

It is important to be aware that the PBG does not act as a filter that would yield only a relative enhancement; the PBG gives rise to an absolute enhancement that could be profitably implemented for low-threshold lasing. The inhibition of the spontaneous emission could be used to favor the stimulated emission in an x-ray lasing system. 


\section{References}

[1] E.M. Purcell, Phys. Rev. 69, 681 (1946).

[2] J. P. Dowling and C. M. Bowden, Phys. A. 46, 612 (1992).

[3] M. Scalora, J. P. Dowling, M. Tocci, M. J . Bloemer, C. M. Bowden, and J. W. Hauss, Appl. Phys. B. 60, S57 (1995).

[4] M. D. Tocci, M. Scalora, M. J. Bloemer, J. P. Dowling, and C. M. Bowden, Phys. Rev. A 53, 2799 (1996).

[5] A. V. Medvedev, N. A.Feoktiskov, A. B. Pevtsov, and V. G. Golubev, Semiconductors 39, 1356 (2005).

[6] H. Yokoyama, Science 256, 66 (1992).

[7] C. Sibilia, I. S. Nefedov, M. Scarola, and M. Bertolli, J. Opt. Soc. Am. B 15, 1947 (1998).

[8] J-M. André and R. Barchewitz, Recent. Res. Devel. Optics 2, 645 (2002).

[9] P. Jonnard, J-M. André, C. Bonnelle, F. Bridou, and B. Pardo, Phys. Rev. A. 68, 032505 (2003).

[10] J-M. André, P. Jonnard, and B. Pardo, Phys. Rev. A. 70, 012503 (2004).

[11] P. Jonnard, J.-M. André, C. Bonnelle, F. Bridou, B. Pardo, Appl. Phys. Lett. 81, 1524 (2002).

[12] A. Yariv and P. Yeh, J. opt. Soc. Am 67, 438 (1997).

[13] J. M. Bendickson, J. P. Dowling, and M. Scalora, Phys. Rev. E 53, 4107 (1996).

[14] J.P. Dowling, IEE Proc. Optoelectron. 145, 420 (1998).

[15] J-M. André and P. Jonnard, J. Mod. Opt. 56, 1562 (2009).

[16] B. Pardo, T. Megademini, and J-M. André, Revue Phys. Appl. 23, 1579 (1988).

[17] P. Yeh, Optical Waves in Layered Media, (Wiley, New York, 1988), Chap.3.

[18] J-M. André and B. Pardo, Optics Comm. 66, 249 (1988).

[19] http://henke.lbl.gov/optical_constants/

[20] J. L. Campbell and T. Papp, At. Data Nucl. Data Tables 77, 1 (2001).

[21] I. Alvarado-Rodriguez, P. Halevi and A. S. Sanchez, Phys. Rev. E 63, 056613 (2001).

[22] J. R. Zurita-Sanchez, A. S. Sanchez, P. Halelevi, Phys. Rev. E 66, 046613 (2001).

[23] P. Haveli and A. S. Sanchez, Optics Comm. 251, 109 (2005).

[24] R. Benbalagh, J.-M. André, R. Barchewitz, P. Jonnard, G. Julié, L. Mollard, G. Rolland,

C. Rémond, P. Troussel, R. Marmoret, E. O. Filatova,, Nucl. Instrum. Methods A 541, 590 (2005).

[25] J. D. Jackson, Classical Electrodynamics, $2^{\text {nd }}$. ed. (Wiley, New York, 1975), Chap.6. 
[26] C.-T. Tai, Dyadic Green Functions in Electromagnetic Theory, $2^{\text {nd }}$. ed. (IEEE, New York, 1994). 


\section{Appendix}

The power radiated by the current $\mathbf{J}(\mathbf{r}, \mathrm{t})$ contained in the volume $\mathrm{V}$ and associated with the radiating dipole can be written [25] :

$$
P(t)=\frac{1}{c} \int_{v} \mathbf{J}(\mathbf{r}, t) \cdot \frac{\partial \mathbf{A}(\mathbf{r}, t)}{\partial t} d V
$$

Since the potential $\mathbf{A}(\mathbf{r}, \mathrm{t})$ can be expressed via the dyadic propagator $\overrightarrow{\mathbf{D}}(\mathbf{r}, t ; \mathbf{R}, \tau)$ which obeys the homogeneous wave Eq. (22), as follows [26] :

$$
\mathbf{A}(\mathbf{r}, t)=\frac{4 \pi}{c} \int_{-\infty}^{t} d \tau \int_{V} d V \overrightarrow{\mathbf{D}}(\mathbf{r}, t ; \mathbf{R}, \tau) . \mathbf{J}(\mathbf{r}, t)
$$

and since $\overrightarrow{\mathbf{D}}(\mathbf{r}, \mathrm{t} ; \mathbf{R}, \tau)$ can be expanded in the NMs a(r) by :

$$
\overrightarrow{\mathbf{D}}(\mathbf{r}, t ; \mathbf{R}, \tau)=c^{2} \int(t-\tau) \sin c\left[\omega_{k}(t-\tau)\right] \mathbf{a}_{\mathbf{k}}^{*}(\mathbf{R}) \mathbf{a}_{\mathbf{k}}(\mathbf{r}) d \mathbf{k}
$$

where sinc stands for the cardinal sine, the total energy $W(T)=\int_{-\infty}^{T} P(t) d t$ is given by :

$$
W(T)=\pi \int d \mathbf{k}\left|\int_{-\infty}^{T} d t \int_{V} d V \mathbf{J}(\mathbf{r}, t) \cdot \mathbf{a}_{\mathbf{k}}(\mathbf{r}) \exp \left(-i \omega_{\mathbf{k}} t\right)\right|^{2}
$$

Assuming that the current density is given by Eq. (3), the total energy is in this case :

$$
W(t)=\pi \Omega^{2} \mu^{2} \int\left|\mathbf{a}_{\mathbf{k}}(\mathbf{R}) \cdot \vec{\mu}\right|^{2}\left(\frac{t}{2}\right)^{2} \sin c^{2}\left[\left(\Omega-\omega_{\mathbf{k}}\right) \frac{t}{2}\right] d^{3} \mathbf{k}
$$

Eq. (4) is deduced from Eq. (A.4) by considering the steady-state rate of emission power :

$$
P(t \rightarrow \infty)=\lim _{t \rightarrow \infty} \frac{d W(t)}{d t}
$$

